\title{
Emisión teórica de metano en tres sistemas de invernada para engorda de ganado en Argentina
}

\author{
Hugo von Bernard ${ }^{1}$, Virginia Vilarino ${ }^{2}$ y Gervasio Piñeiro 3 \\ ${ }^{1}$ Escuela para graduados (EPG), Facultad de Agronomía, Universidad de Buenos Aires (UBA) y \\ Consejo Nacional de Investigaciones Científicas y Técnicas (CONICET), Av. San Martín 4453, CP:C1417DSE, \\ Buenos Aires, Argentina. ${ }^{2}$ Consultor privado, Av. Córdoba 1470, Piso 6․ Departamento L, CP 1055, Buenos Aires, \\ Argentina. ${ }^{3}$ Instituto de Investigaciones Fisiológicas y Ecológicas Vinculadas a la Agricultura (IFEVA), \\ Facultad de Agronomía, UBA y CONICET.
}

\begin{abstract}
H. von Bernard, V. Vilarino, and G. Piñeiro. 2007. Theoretical emission of methane in three systems to fatten cattle in Argentina. Cien. Inv. Agr. 34(2):121-129. In this study, the total annual and theoretical emission of methane $\left(\mathrm{CH}_{4}\right)$ was estimated for cattle subjected to three winter fattening systems used in Argentina. These were extensive, semi-intensive and intensive fattening systems. The estimated annual emission was expressed as animal, live weight, cattle area and surface area used. The crude energy (CE) consumption was estimated on the basis of the method proposed by the Intergovernmental Panel for the Climatic Change (IPCC), and also following the cow equivalent (cE) adapted to the Argentine conditions in this study. To calculate the $\mathrm{cE}$, the annual consumption of concentrates and forage of all the animals present in the farm was considered. At the same time, the digestibility of the consumed foods and the conversion factor of crude energy to methane suggested by IPCC were used. To estimate $\mathrm{CH}_{4}$ emission from the $\mathrm{CE}$ consumptions, the conversion $\mathrm{CE}$ factor $(\mathrm{Ym})$ suggested by the IPCC, and a second factor (proposed in this study) were used. Independently of the method used to estimate $\mathrm{CH}_{4}$ emission, the results obtained indicate that methane emission, expressed as animal and live weight produced, was higher in the extensive systems; however, the estimation of $\mathrm{CH}_{4}$ emission was greater in the intensive systems in terms of cattle area and used surface area. These results are important considering that eventually the emission of $\mathrm{CH}_{4}$ will be punished as a means to prevent the development of the greenhouse effect. Thus, it will be necessary to define how to measure and express these results. Today, some people suggest the intensification of animal production in order to reduce the emission of $\mathrm{CH}_{4}$ per animal without considering the emission per cattle area or utilized area and without considering the origin of the livestock feed.
\end{abstract}

Key words: Bovine, cattle, fattening systems, methane, methane emission by animal.

\section{Introducción}

Los gases que emiten los bovinos, por ejemplo anhídrido carbónico $\left(\mathrm{CO}_{2}\right)$ y metano $\left(\mathrm{CH}_{4}\right)$ se diferencian en cantidad, por su efecto invernadero (EI) y por la distribución espacial de sus efectos. El $\mathrm{CH}_{4}$ se genera por la degradación de los carbohidratos presentes en los alimentos y la descomposición anaeróbicas

Recibido 06 de noviembre 2006. Aceptado 01 de mayo 2007.

${ }^{1}$ Dirigir correspondencia a H.von Bernard: vonberna@agro.uba.ar de las excretas (Müller y Bartsch, 1999; de Lima, 2002; McGinn et al., 2004). Su determinación se realiza a través del uso de máscaras (Johnson y Johnson 1995) o se estiman por medio del uso de fórmulas y coeficientes derivados de la energía bruta (EB) consumida por los animales (Berra et al., 1999; Sharma et al., 2002; Ulyatt et al., 2002). La emisión de metano se estima generalmente según las fórmulas del IPCC (IPCC 1996) o del equivalente vaca (EV). El EV considera los requerimientos de mantenimiento y producción de un animal y permite realizar comparaciones entre diferentes categorías de 
animales y balances forrajeros (Cocimano et al., 1983).

En Argentina, la carne bovina es un producto no diferenciado (commodity) cuyo precio tiende al costo de producción. Debido a que la emisión de $\mathrm{CH}_{4}$ representa una pérdida de energía en los sistemas ganaderos, se intenta minimizarla a través de una mayor concentración energética en la alimentación (sistemas más intensivos de invernada) (Palliser y Woodward, 2002). En ese contexto, mientras el ganadero busca su sustentabilidad económica e intensifica la producción, la sociedad persigue la sustentabilidad social (Naredo, 2006). Sin embargo, como la sociedad no genera incentivos que fomenten la sustentabilidad ambiental de las actividades productivas, el productor se abstiene de hacerlo (Aylward et al., 1999; Ghida Daza, 2003).

La invernada vacuna argentina se realiza normalmente sobre pasturas perennes y cultivos forrajeros anuales, con o sin suplementación estratégica de energía por otros medios. Sin embargo, en estos últimos años en respuesta a una baja relativa del precio de los suplementos energéticos con respecto a la carne, la invernada se intensificó. La intensificación de la invernada lleva al encierre de bovinos en sistemas intensivos (feedlots), al aumento de la calidad y cantidad de la dieta animal y a una reducción de la emisión total de $\mathrm{CH}_{4}$. Según la metodología propuesta por el IPCC (1996), se emitieron 2.577.346 ton de $\mathrm{CH}_{4}$ en 1997 en Argentina, siendo 1,4 y $6,0 \%$ inferiores a las emisiones de 1990 y 1994, respectivamente. Esto se atribuyó a una supuesta mayor eficiencia en el manejo ambiental (Berra y Finster, 2003).

La contaminación es directamente criticada por la comunidad debido a que afecta negativamente la calidad de vida. Además, la contaminación también preocupa a las Organizaciones no Gubernamentales (ONGs) por cuanto es un riesgo contra la sustentabilidad ambiental global. No obstante, en los sistemas de producción intensivos casi todo el alimento consumido proviene de lugares situados a cientos o miles de kilómetros. Pocos trabajos sobre emisión de $\mathrm{CH}_{4}$ por bovinos reconocen esta situación. Esto involucra gasto energético en el transporte de alimento y deja sin utilizar una gran cantidad de fibra en forma de rastrojos. Tampoco se menciona que la concentración animal hace que la emisión de gases tenga un mayor efecto invernadero (EI) por unidad de superficie en comparación con los sistemas pastoriles.

El objetivo de este trabajo fue estimar la cantidad anual teórica de $\mathrm{CH}_{4}$ emitido en tres sistemas de invernada utilizados en Argentina. Al mismo tiempo se analiza su incidencia por animal, peso vivo producido, superficie ganadera y superficie utilizada.

\section{Materiales y métodos}

La determinación de $\mathrm{CH}_{4}$ emitido por los bovinos se realizó sobre la base de la energía bruta (EB) consumida. Para ello, se usó la fórmula del IPCC (1996) y el equivalente vaca (EV) (Cocimano et al., 1983).

La determinación de la cantidad de $\mathrm{CH}_{4}$ emitido se calculó a través de un factor de emisión de $\mathrm{CH}_{4}(\mathrm{Ym})$ que relacionó la digestibilidad del alimento con la cantidad de $\mathrm{CH}_{4}$ producido. En este trabajo se utilizó dos tipos de Ym, aquellos formulados por el IPCC (1996) e índices desarrollados por los autores, teniendo en cuenta la digestibilidad del alimento en los distintos sistemas de invernada utilizados en Argentina y en forma similar a lo propuesto por Boadi et al.(2001).

Los valores de Ym usados por el IPCC fueron: $0,065(6,5 \%)$ para los sistemas pastoriles y $0,03(3,0 \%)$ para los sistemas intensivos de alimentación. Esto tiene importancia cuando se determina la emisión de metano por parte de la ganadería vacuna de un establecimiento o de un país.

La pérdida del $6 \%$ de la EB consumida como $\mathrm{CH}_{4}$ es un valor alto (Westberg et al., 2001; Mangino, 2003). Por lo tanto, basados en la mayor digestibilidad de la dieta de invernada con respecto a la de cría, se propuso un Ym de $0,03(3,0 \%)$ para los sistemas intensivos (feedlots) al igual que el valor Ym propuesto por IPCC $(4,0 \%)$. Para los sistemas semiintensivos y extensivos se propuso $0,04(4,0 \%)$ y $0,05(5,0 \%)$, respectivamente (Cuadro 1$)$. 
Cuadro 1. Digestibilidad del alimento y factores usados (Ym) para estimar la emisión de metano $\left(\mathrm{CH}_{4}\right)$ por el ganado bovino en tres tipos de invernadas en Argentina.

Table 1. Food digestibility and factor $(\mathrm{Ym})$ use to estimate methane $\left(\mathrm{CH}_{4}\right)$ emission produced by bovine cattle in three types of winter pasture in Argentina.

\begin{tabular}{lcc}
\hline $\begin{array}{l}\text { Tipo de } \\
\text { invernada }\end{array}$ & $\begin{array}{c}\text { Digestibilidad del alimento } \\
\%\end{array}$ & $\mathrm{Ym}^{2}$ de $\mathrm{CH}_{4}$ \\
\hline Extensiva & 60 & 0,05 \\
Semi - intensiva & 70 & 0,04 \\
Intensiva & 80 & 0,03 \\
\hline
\end{tabular}

${ }^{1}$ Invernada extensiva, animales alimentados solamente a forraje. Invernada semi-intensiva, animales alimentados un $90 \%$ con forraje y el $10 \%$ restante con concentrados. Invernada intensiva, animales alimentados en un $90 \%$ con concentrados y un $10 \%$ con forraje. En el año, se consideran tres ciclos de cerca de 120 días.

${ }^{2} \mathrm{Ym}$, factor de emisión, relaciona el consumo de energía bruta del alimento con la emisión de $\mathrm{CH}_{4}$.

${ }^{1}$ Extensive fattening system, animals were fed only by forage. Semi-intensive fattening system, animals were fed with $90 \%$ forage and $10 \%$ concentrate. Intensive fattening system, animals were fed with $90 \%$ concentrate and $10 \%$ forage. In one year, three cycles of 120 days take place.

${ }^{2} Y m$, emission factor that relates the gross energy consumed to $\mathrm{CH}_{4}$ emission.

\section{Tipos de invernada considerados en este estudio}

Las invernadas bovinas argentinas son descritas como sistemas de producción extensivos, semi-intensivos e intensivos de acuerdo con la cantidad y calidad de alimentos que reciben los animales (Torroba, 1988). En este trabajo, se asumió que: 1 . Todos los campos se encuentran en la misma zona, 2. Los animales se venden terminados con 4 a $8 \mathrm{~mm}$ de grasa subcutánea y 3. Ningún sistema presentó mortandad. Por otra parte, se asumió que la cantidad y calidad del alimento que se les entregó satisfizo las necesidades para la ganancia diaria de peso estipulada.

En la invernada extensiva los animales obtuvieron más del $90 \%$ de su alimento de pastizales, pasturas y verdeos. Los verdeos son forraje sembrados y usualmente consumido en menos de ocho meses de invierno, compuestos por algún cereal en pie como avena, cebada o centeno. El 10\% restante lo obtuvieron de reservas forrajeras (rollos, fardos) y/o rastrojos de cosecha (Torroba, 1988). En esta modelización, se estudió el consumo anual de 1200 terneros de raza británica de tamaño medio, entrando sistema con $180 \mathrm{~kg}$ de peso vivo (PV) y vendidos 18 meses más tarde con $460 \mathrm{~kg}$. Existió superposición de categorías en parte del año. Para ello se necesitaron 855 ha de pasturas y 190 ha de verdeos (VI) que sólo se usaron 9 de los 12 meses del año. En total se consideraron 998 ha en este modelo. En el invierno, los animales se suplementaron con $200 \mathrm{~kg}$ de materia seca (MS, seca a $60^{\circ} \mathrm{C}$ hasta obtener un peso constante) de rollos obtenidos en el propio campo (von Bernard, 2006).

En la invernada semi-intensiva, los animales recibieron el $10 \%$ de su alimento como granos, o restos de cosecha (Torroba, 1988). En este modelo, todos los años se compraron 2200 terneros de raza británica de tamaño medio de $180 \mathrm{~kg}$ de PV. Se los invernó durante 14 meses, hasta un peso de $420 \mathrm{~kg}$. Por lo tanto, existió superposición de categorías durante 2 meses. Este campo posee 855 ha de pasturas, a lo que se le agregan 190 ha de verdeos que sólo se usan 9 de los 12 meses del año, equivalente a 112 ha de maíz. En total 1110 ha. Los 2200 animales se suplementan a razón de $4 \mathrm{~kg} \cdot$ día $^{-1}$ de maíz durante los últimos 120 días previos a la faena (von Bernard, 2006).

En la invernada intensiva los animales recibieron $90 \%$ de su alimento en concentrados energéticos y $10 \%$ como forraje (Torroba, 1988). En este modelo, se partió de un establecimiento de 5 ha con corrales para realizar el inverne. Se dispuso de una hectárea con una laguna de decantación en tres circuitos de 1000 terneros de raza británica de tamaño medio por vez. El PV inicial fue de $180 \mathrm{~kg}$. Se vendieron como terneros bolita que son animales para marcado interno, no exportables, engordado en sistema intensivo hasta 280 a $345 \mathrm{~kg}$ de PV. Para cubrir las necesidad total de maíz (1.828 ton) se necesitaron 261 ha que, como se usaron solo 9 meses en el año, correspondieron a 195 ha·año ${ }^{-1}$. El $10 \%$ del alimento restante se proporcionó en rollos que correspondieron 28 ha, ya que $203.173 \mathrm{~kg}$ MS de forraje por $600 \mathrm{~kg}$ MS por rollo totalizan 338 rollos. Considerando que se producen 12 rollos por ha $\cdot{ }^{2} \tilde{n}^{-1}$, se necesitaron 28 ha con este propósito. En total fueron 230 ha, compuestas por 195 ha de maíz, 28 ha de forraje para rollos y 6 ha de instalaciones generales (von Bernard, 2006). 
Si la emisión de $\mathrm{CH}_{4}$ ocurre donde se obtuvieron los alimentos, su emisión se debería evaluar respecto de esa superficie. En cambio, si la emisión de $\mathrm{CH}_{4}$ se realiza lejos de la superficie ganadera se deberá considerar la superficie real ocupada. En este caso, la emisión de gases de una gran superficie se ha concentrado en un solo lugar.

Estimación de la energía bruta y cálculo de la emisión de $\mathrm{CH}_{4}$ bajo distintos niveles de intensificación

El IPCC (1996) utiliza para el cálculo de la energía bruta (EB) consumida $\left(\mathrm{MJ} \cdot \mathrm{dí}^{\mathrm{a}}{ }^{-1}\right)$, empleando la ecuación (1):

$E B(M J /$ dia $)=\left[\frac{\left(E N_{m}+E N_{a l}\right)}{E \overline{E N}}+\frac{E N_{c}}{E N_{c} E D}\right] \cdot 100 / \% D E$

dónde EN es energía neta, ED es energía digestible (ED = EB- energía en las fecas $(\mathrm{EF})$ ), $\mathrm{EN}_{\mathrm{m}}$ es energía neta de mantenimiento, $\mathrm{EN}_{\mathrm{al}}$ es energía neta del alimento, $\mathrm{EN}_{\mathrm{c}}$ es energía neta para el crecimiento. La EN corresponde a la energía metabolizable (EM) menos la perdida por calor, siendo la EM = ED-energía en la orina (EU).

De acuerdo con esta fórmula se estimó la energía bruta (EB) requerida por los animales, en función de la $\mathrm{EN}_{\mathrm{c}}$ necesaria para alcanzar la ganancia diaria de peso (GDP) $\left(\mathrm{kg}^{\cdot} \mathrm{dí}^{-1}\right)$ especificada en los distintos tipos de invernada. El consumo de EB de todos los animales que se encuentran en cada sistema de invernada se evaluó como equivalente vaca (EV) (Cocimano et al., 1983) (Cuadro 2). En este cálculo, se consideró a todos los animales, su peso en cada momento del año, su ganancia diaria de peso (GDP) en distintos momentos del año y el forraje más suplemento que los animales deben consumir para alcanzar esas GDP. Los EV anuales consumidos en cada sistema de invernada se transformaron en Mcal de energía metabolizable (EM), al multiplicarlos por su equivalente energético 18,54 Mcal de EM por

Cuadro 2. Consumo de alimentos y emisión de metano $\left(\mathrm{CH}_{4}\right)$ estimado en ganado bovino en diferentes tipos de invernadas en Argentina.

Table 2. Food consumption and methane $\left(\mathrm{CH}_{4}\right)$ emissions for each type of winter fattening system used in Argentina.

\begin{tabular}{|c|c|c|c|}
\hline Parámetros ${ }^{1}$ & extensiva & $\begin{array}{l}\text { Tipo de invernada } \\
\text { semi-intensiva }\end{array}$ & intensiva \\
\hline Tiempo de invernada, días & 563 & 440 & $3 * 121$ \\
\hline Consumo equivalente vaca (EV) & 593.148 & 788.106 & 331.740 \\
\hline Energia metabolizable de forraje (EM), Mcal & 10.996 .964 & 11.637 .725 & 1.011 .260 \\
\hline Materia seca total de granos (MS), $\mathrm{kg}$ & 0 & 929.300 & 1.606 .000 \\
\hline Energía metabolizable consumida (EM), Mcal & 10.996 .964 & 14.611 .485 & 6.150 .460 \\
\hline Energía metabolizable de granos (EM), Mcal & 0 & 2.973 .760 & 5.139 .200 \\
\hline Materia seca total de granos (MS), kg & 0 & 929.300 & 1.606 .000 \\
\hline Materia seca total de forraje (MS), $\mathrm{kg}$ & 4.781 .288 & 5.059 .880 & 439.678 \\
\hline Materia seca consumida (MS), $\mathrm{kg}^{-h^{-1}}$ & 4.790 & 5.070 & 377.446 \\
\hline Energía bruta (EB), Mcal·año ${ }^{-1}$ & 19.158.474 & 25.455 .549 & 9.375 .701 \\
\hline Energía bruta (EB), Mj·año ${ }^{-1}$ & 80.159 .054 & 106.506 .018 & 39.227 .931 \\
\hline $\mathrm{CH}_{4}$ total emitido, $\mathrm{kg}$ & 95.787 & 127.261 & 22.147 \\
\hline
\end{tabular}

${ }^{1}$ Valores según von Bernard (2006). EV, considera los requerimientos de mantenimiento y producción de un animal y permitió realizar comparaciones entre diferentes categorías de animales y balances forrajeros. Equivale a 18,54 Mcal de EM [EM = EB-EF (materia fecal)EU (orina)]. MS, alimento seco a $60^{\circ} \mathrm{C}$ hasta peso constante. $\mathrm{EB}=$ energía total del alimento medido por bomba calorimétrica.

${ }^{2}$ Invernada extensiva, animales alimentados solamente a forraje. Invernada semi-intensiva, animales alimentados un $90 \%$ con forraje y el 10 $\%$ restante con concentrados. Invernada intensiva, animales alimentados en un $90 \%$ con concentrados y un $10 \%$ con forraje. Se consideran tres ciclos, de cerca de 120 días, en el año.

${ }^{\prime}$ Values were according to von Bernard (2006). cE, consumption of cow equivalent, considering the requirement for maintenance and production of one animal that allowed a comparison to be made between categories and different forage balances. ME is equivalent to $18.54 \mathrm{Mcal}\left[\mathrm{EM}=\mathrm{CE}-\mathrm{FE}\right.$ (fecal energy)-UE (urine energy)]. DM, food dries at $60^{\circ} \mathrm{C}$ until to obtain constant weight. CE $=$ total energy of food determined by means of a calorimetric pump.

${ }^{2}$ Extensive fattening system, animals were fed only by forage. Semi-intensive fattening system, animals were fed with $90 \%$ forage and $10 \%$ concentrate. Intensive fattening system, animals were fed with $90 \%$ concentrate and $10 \%$ forage. In one year, three cycles of 120 days take place. 
EV (Cocimano et al., 1983). Para convertir las Mcal de EM en Mcal de EB se dividió el valor de la EM por la digestibilidad del alimento considerado y por 0,82 que representó la proporción que no se pierde como gases y orina. La transformación a Mjoules (MJ) de las Mcal de EB consumidas se obtuvo multiplicando cada Mcal de EB por 4,184 (Cuadro 2).

A partir de los MJ de la $E B$ consumida se estimó las emisiones de $\mathrm{CH}_{4}$ usando la metodología y los valores recomendados por el IPCC (1996) y los valores previamente sugeridos por los autores (Cuadro 1). Los MJ de $E B$ consumida se convirtieron en $\mathrm{CH}_{4}$ de acuerdo con la ecuación (2) del IPCC:

Emisiones de $\mathrm{CH}_{4}=\frac{\left(\text { Consumo EB }\left(\text { MJ.día }^{-1}\right) \times \mathrm{Ym} \times 365 \text { (días.año }^{-1}\right)}{55,65 \mathrm{MJ}^{-1} \text { ) }}$ $\left(\mathrm{kg} \cdot \mathrm{año}^{-1}\right) \quad \frac{55,65 \mathrm{MJ} \cdot \mathrm{kg}^{-1} \mathrm{de} \mathrm{CH} 4}{}$

En esta ecuación, Ym, fue el factor de conversión de la EB consumida en $\mathrm{CH}_{4}$. Este valor varió entre el 3,0 y el 6,5\% de lo consumido (Johnson y Johnson, 1995; IPCC, 1996; Mangino, 2003). Para calcular las emisiones de $\mathrm{CH}_{4}$ el consumo anual medido en $\mathrm{MJ}$ se dividió por 55,65 $\mathrm{MJ} \cdot \mathrm{kg}^{-1}$ que correspondieron a cada $\mathrm{kg}$ de $\mathrm{CH}_{4}$ emitido.

De la emisión anual de $\mathrm{CH}_{4}$ de cada sistema se derivó el peso en $\mathrm{kg}$ de $\mathrm{CH}_{4}$ emitidos por $\mathrm{kg}$ de PV producido, por cabeza, por superficie ganadera y por superficie utilizada. La superficie ganadera se definió como la superficie total necesaria para proveer de alimentos a los animales, mientras que la superficie utilizada hace referencia a la superficie donde se encuentran los animales, independientemente de donde se obtuvo los alimentos.

En la práctica, al valor de $\mathrm{CH}_{4}$ emitido directamente por los rumiantes, se le suma la emisión de un $\mathrm{kg}$ de $\mathrm{CH}_{4}$ por animal y por año, como consecuencia de la emisión por parte de la material fecal de cada animal (Berra et al., 1999; Berra y Finster, 2003). Si bien se estimó la cantidad de excretas producidas por animal teniendo en cuenta las características del alimento, consumoy digestibilidad, sobre labase de su MS, hay que tener en cuenta que el $\mathrm{CH}_{4}$ del estiércol se produce por la descomposición anaeróbica del mismo (Hansen et al., 2002).
Por lo tanto, un $\mathrm{kg} \mathrm{de} \mathrm{CH}_{4}$ por animal y por año, en todos los sistemas de invernada analizados, supuso asumir que la casi totalidad del estiércol quedó depositado directamente en las pasturas, descomponiéndose aeróbicamente y produciendo menos $\mathrm{CH}_{4}$ que las heces húmedas del sistema intensivo de alimentación.

\section{Resultados y discusión}

En este trabajo se determinó el consumo teórico de equivalente vaca (EV), la ganancia diaria de peso (GDP) en diferentes invernadas, extensivas, semi-intensivas e intensivas, en Argentina. Por medio del EV, se calculó el consumo de EB (Cuadro 2). De acuerdo con estos resultados el mayor consumo anual lo presentó la invernada semi-intensiva, seguida por la invernada extensiva e intensiva.

Los valores de Ym propuestos en este estudio se indican en el Cuadro 1. El Ym según lo propuesto por IPCC (1996) para la invernada extensiva y semi-intensiva fue $6 \%$ de lo consumido, y en la invernada intensiva fue el 3\% (Cuadro 3). En el Cuadro 2 se presenta el consumo de alimentos y la emisión estimada de $\mathrm{CH}_{4}$ por el ganado bovino en diferentes tipos de invernadas de Argentina.

La emisión teórica de $\mathrm{CH}_{4}$, partiendo de la metodología de cálculo del EV para la determinación de la EB consumida, se presenta en el Cuadro 3. Para la transformación de los $\mathrm{MJ}$ de $\mathrm{EB}$ en $\mathrm{CH}_{4}$ se utilizaron los Ym del IPCC y los propuestos por los autores. Se destaca la influencia del valor del Ym sobre la estimación de la emisión de $\mathrm{CH}_{4}$.

Si se toma como $\mathrm{Ym}$ de $\mathrm{CH}_{4}$ los valores propuestos previamente por los autores, 3, 4 y $5 \%$ para las invernadas intensiva, semiintensiva y extensiva, respectivamente (Cuadro 1) se obtendrán los resultados que se muestran en los Cuadros 3 y 4 . En todos los casos, en las invernadas pastoriles, extensivas y semiintensivas, la emisión de $\mathrm{CH}_{4}$ estimada fue menor que la propuesta por el IPCC (1996).

En el Cuadro 4 se utilizaron los valores resultantes de la fórmula (1) del IPCC (1996) por un lado y del EV por el otro para el cálculo de 
Cuadro 3. Emisión de metano $\left(\mathrm{CH}_{4}\right)$ estimado para diferentes tipos de invernadas en Argentina, partiendo del consumo determinado por el equivalente vaca (EV), empleando el factor de conversión (Ym) propuesto por el Intergovernmental Panel on Climate Change (IPCC) y el factor propuesto en este trabajo.

Table 3. Estimated methane $\left(\mathrm{CH}_{4}\right)$ emissions for different fattening systems used in Argentina based on animal consumption determined by cow equivalent ( $c E)$, using the conversion factors $(Y m)$ suggested by the Intergovernmental Panel on Climate Change (IPCC) and the factors proposed in this study.

\begin{tabular}{|c|c|c|c|c|c|}
\hline \multirow[b]{2}{*}{ Tipo de invernada ${ }^{1}$} & \multicolumn{5}{|c|}{ Emisión de metano $\left(\mathrm{CH}_{4}\right)$} \\
\hline & $\begin{array}{l}\text { Total } \\
\text { ton }\end{array}$ & $\begin{array}{l}\text { Por kilo producido } \\
\mathrm{g}\end{array}$ & $\begin{array}{l}\text { Por animal } \\
\mathrm{kg}\end{array}$ & $\begin{array}{c}\text { Por hectárea } \\
\text { utilizada } \\
\text { kg }\end{array}$ & $\begin{array}{c}\text { Por hectárea } \\
\text { ganadera } \\
\text { kg }\end{array}$ \\
\hline \multicolumn{6}{|c|}{ Estimado según el factor de emisión (Ym) del IPCC (1996): } \\
\hline Extensiva & 95,78 & 280 & 44,35 & 96 & 96 \\
\hline Semi-intensiva & 127,26 & 240 & 44,50 & 115 & 115 \\
\hline Intensiva & 22,14 & 60 & 22,15 & 3691 & 615 \\
\hline \multicolumn{6}{|c|}{ Estimado según el factor de emisión (Ym) propuesto en este trabajo: } \\
\hline Extensiva & 74,18 & 220 & 34,34 & 74 & 74 \\
\hline Semi-intensiva & 79,41 & 150 & 27,77 & 72 & 72 \\
\hline Intensiva & 22,14 & 60 & 22,15 & 3691 & 615 \\
\hline
\end{tabular}

${ }^{1}$ Valores según von Bernard (2006). Invernada extensiva, animales alimentados solamente a forraje. Invernada semi-intensiva, animales alimentados un $90 \%$ con forraje y el $10 \%$ restante con concentrados. Invernada intensiva, animales alimentados en un $90 \%$ con concentrados y un $10 \%$ con forraje. Se consideran tres ciclos, de cerca de 120 días, en el año. Se consideró los valores de transformación de la EB en $\mathrm{CH}_{4}$ del IPCC (1996).

${ }^{I}$ Values were according to von Bernard (2006). Extensive fattening system, animals were fed only by forage. Semi-intensive fattening system, animals were fed with $90 \%$ forage and $10 \%$ concentrate. Intensive fattening system, animals are fed with $90 \%$ concentrate and $10 \%$ forage. In one year, three cycles of 120 days take place. Values of forage energy transformation in methane were used according to the IPCC (1996).

la EB consumida en cada una de las invernadas analizadas. En ambos casos se ponderaron por los Ym del IPCC y los propuestos por los autores.

De la comparación de los Cuadros 3 y 4 se desprende que: a. La estimación de la emisión total anual de $\mathrm{CH}_{4}$ fue mayor usando los $\mathrm{Ym}$ del IPCC y menor con los valores propuestos por los autores, excepto en la invernada intensiva. En este último caso, se usó el mismo valor Ym. b. Independientemente del Ym usado, el sistema de invernada que mayor cantidad total de $\mathrm{CH}_{4}$ emitió fue el semi-intensivo. c. El sistema de invernada que menos $\mathrm{CH}_{4}$ emitió por animal y por $\mathrm{kg}$ de producto fue la invernada intensiva. d. El sistema que menos $\mathrm{CH}_{4}$ emitió por superficie ganadera fue el sistema de pastoreo extensivo.

Por lo tanto, mientras la emisión de $\mathrm{CH}_{4}$ por peso vivo producido y por animal aumentó a medida que se utilizó un sistema de producción extensivo, pero se redujo la emisión estimada por hectárea ganadera y superficie utilizada. Estas diferencias son especialmente importantes en el caso de los sistemas intensivos donde la superficie ganadera y la superficie utilizada no siempre concuerdan en el espacio y en el tiempo. En algunos casos, la producción y el consumo de los alimentos que reciben los animales está separada por cientos o miles de kilómetros. Como el engorde intensivo de animales emite una gran cantidad de $\mathrm{CH}_{4}$ por superficie ganadera utilizada, si este $\mathrm{CH}_{4}$ fue fijado como $\mathrm{CO}_{2}$ en otros países, los animales actúan como contaminadores ambientales locales.

Cabe destacar las diferencias en la emisión total de $\mathrm{CH}_{4}$, dependiendo del método que se utilice para determinarla, para los sistemas extensivo y semi-extensivo, los valores máximos de emisión de $\mathrm{CH}_{4}$ se dan cuando se utiliza la metodología IPCC para el cálculo de la EB consumida y los $\mathrm{Ym}$ en $\mathrm{CH}_{4}$ del IPCC (1996). En cambio, cuando se utiliza la metodología del EV para la determinación del consumo adaptado a las condiciones locales y los Ym en $\mathrm{CH}_{4}$ propuestos por los autores, las emisiones muestran valores más bajos.

De acuerdo con los diferentes $\mathrm{Ym}$ de $\mathrm{CH}_{4}$, las diferencias entre los valores mínimos y 
Cuadro 4. Estimación de la emisión anual de metano $\left(\mathrm{CH}_{4}\right)$ por bovino según distintos metodologías de análisis.

Table 4. Estimation of the annual emission of methane $\left(\mathrm{CH}_{4}\right)$ by bovine cattle according to different methods of analysis.

\begin{tabular}{|c|c|c|c|}
\hline \multirow[t]{2}{*}{$\begin{array}{l}\text { Factor de } \\
\text { conversión }\end{array}$} & \multicolumn{3}{|c|}{$\begin{array}{l}\text { Emisión estimada de } \mathrm{kg} \text { de } \mathrm{CH}_{4} \text { por animal } \mathrm{anno}^{-1} \\
\text { en los siguientes tipos de invernada }\end{array}$} \\
\hline & Extensiva & Semi-intensiva & Intensiva \\
\hline \multicolumn{4}{|l|}{ Consumo IPCC ${ }^{2}$} \\
\hline Ym IPCC & 55,42 & 46,29 & 17,71 \\
\hline Ym propuestos & 42,63 & 28,48 & 17,71 \\
\hline \multicolumn{4}{|l|}{ Consumo EV $V^{2}$} \\
\hline Ym IPCC & 43,35 & 43,50 & 21,15 \\
\hline Ym propuestos & 33,34 & 26,77 & 21,15 \\
\hline \multicolumn{4}{|c|}{$\begin{array}{l}{ }^{1} \text { Invernada extensiva, animales alimentados solamente a forraje. Invernada semi-intensiva, animales alimentados un } 90 \% \text { con forraje y el } 10 \\
\% \text { restante con concentrados. Invernada intensiva, animales alimentados en un } 90 \% \text { con concentrados y un } 10 \% \text { con forraje. Se consideran } \\
\text { tres ciclos, de cerca de } 120 \text { días, en el año. Se consideró los valores de transformación de la EB en } \mathrm{CH}_{4} \text { según IPCC (1996). } \\
{ }^{2} \text { Emisión estimada de kg de metano por animal mediante metodología IPCC (1996) para el cálculo de la energía bruta (EB) consumida } \\
\text { [ecuación (1)] y usando los factores de conversión (Ym) de metano propuestos por el IPCC y los propuestos por los autores en la ecuación } \\
\text { (2). En este caso no se consideró el metano eliminado por las excretas. Equivalente vaca (EV), valor que consideró los requerimientos } \\
\text { de mantenimiento y producción de un animal y que permitió realizar comparaciones entre diferentes categorías de animales y balances } \\
\text { forrajeros. Ym, factores que relacionan la digestibilidad del alimento con la emisión de } \mathrm{CH}_{4} \text {. } \\
{ }^{1} \text { Extensive fattening system, animals were fed only by forage. Semi-intensive fattening system, animals were fed with } 90 \% \text { forage and } 10 \% \\
\text { concentrate. Intensive fattening system, animals are fed with } 90 \% \text { concentrate and } 10 \% \text { forage. In one year, three cycles of } 120 \text { days take } \\
\text { place. Values of forage energy transformation in methane were used according to the IPCC (1996). } \\
{ }^{2} \text { Estimated methane emission (kg) per animal by means of the IPCC methodology (1996) for calculating the gross energy and using the } \\
\text { values of methane conversion of the IPCC and the one suggested by the authors. In this case, the methane eliminated does not consider } \\
\text { excretions. }\end{array}$} \\
\hline
\end{tabular}

máximos de emisión de $\mathrm{CH}_{4}$ puede tener una fuerte implicancia al estimar las emisiones totales de $\mathrm{CH}_{4}$ del sector ganadero bovino de un país. En ese caso, habrá que determinar primero la proporción de bovinos que se alimentan sobre: a. pasturas naturales (pastizales), b. pasturas implantadas, c. pasturas implantadas más suplementos energéticos $\mathrm{y} d$. en sistemas productivos intensivos. Con ello se podrá determinar en consumo de EB de cada grupo y luego, con los diversos Ym, determinar la emisión total de $\mathrm{CH}_{4}$ por grupo y por país.

Por lo tanto, los resultados de este trabajo permiten concluir que existen variaciones considerables en la estimación de la cantidad de $\mathrm{CH}_{4}$ teórico total emitido por los animales. Esto depende de la alimentación, la metodología de determinación de la EB consumida y del Ym que se utilice en cada sistema de producción. La estimación del $\mathrm{CH}_{4}$ emitido tendrá cada vez mayor importancia en la medida que se decida castigando la mayor emisión de $\mathrm{CH}_{4}$ como estrategia para prevenir el efecto invernadero (EI) en el mundo.

\section{Resumen}

En este trabajo se modeló la emisión teórica, total y anual de metano $\left(\mathrm{CH}_{4}\right)$ proveniente de bovinos en tres sistemas de invernadas comúnmente empleadas para engorda de ganado vacuno en Argentina. Estos fueron sistemas extensivos, semi-intensivos e intensivos. La emisión anual estimada, se expresó por animal, peso vivo producido, superficie ganadera y superficie utilizada. El consumo de energía bruta (EB) se estimó se acuerdo con el método propuesto por el Panel Intergubernamental para el Cambio Climático (IPCC) y del equivalente vaca (EV) adaptado a las condiciones argentinas en este estudio. Para el cálculo del EV, se tuvo en cuenta el consumo anual de forraje y concentrados de todos los animales presentes en un establecimiento ganadero; como asimismo, la digestibilidad de los alimentos consumidos. Para estimar la emisión de $\mathrm{CH}_{4}$ a partir del consumo de $\mathrm{EB}$, se usó el factor de conversión (Ym) de $\mathrm{EB}$ en $\mathrm{CH}_{4}$ sugerido por IPCC. Además, se usó un segundo factor propuesto en este trabajo. Independiente del método para estimar la emisión de $\mathrm{CH}_{4}$, los 
resultados obtenidos indicaron que la emisión expresada por animal y por peso vivo producido fue mayor en los sistemas de producción extensivos. Sin embargo, la emisión de $\mathrm{CH}_{4}$ estimada por superficie ganadera o superficie utilizada fue mayor en los sistemas intensivos de producción. La importancia de este aserto reside en que eventualmente se sancionará las altas emisiones de $\mathrm{CH}_{4}$ como medida para prevenir el efecto invernadero. Cuando esto suceda habrá que definir, en el caso de la ganadería vacuna, como medir y expresar estos resultados. Actualmente, algunos proponen intensificar la producción animal para reducir la emisión de $\mathrm{CH}_{4}$ por unidad de producto animal, sin considerar la emisión por superficie ganadera o por superficie utilizada y sin considerar el origen de los alimentos.

Palabras clave: Bovinos, emisión de metano, engorda animal, sistemas de invernada.

\section{Literatura citada}

Aylward, B., J. Echeverría, K. Allen, R.Mejías, and I.-T. Porras. 1999. Market and policy incentives for livestock production and watershed protection in arenal, Costa Rica. CREED, Programa de Investigación en Colaboración en Economía del Medio Ambiente, Instituto Internacional de Medio Ambiente y Desarrollo (IIMAD). Working Paper No 25. London, England. www.iied.org/creed. (Consultado en julio 2002).

Berra, G. y L. Finster, 2003. La ganadería argentina y la emisión de gases efecto invernadero. Revista Idia (Argentina) 21:212-215.

Berra, G., L. Finster, E. Castuma y V. Maldonado. 1999. Reducción de Emisiones de Metano Provenientes del Ganado Bovino. Ministerio de Desarrollo Social y Medio Ambiente. Secretaría de Desarrollo Sustentable y Política Ambiental. Argentina. 142 pp.

Boadi, D.A., K.M. Wittenberg, and W.P. McCaughey. 2000. Effects of grain supplementation on methane production of grazing steers using the sulphur (SF6) tracer gas technique. Canadian Journal of Animal Science 82:151-157.

Cocimano, M., A Lange y E. Menvielle. 1983. Equivalencias ganaderas para vacunos de carne y ovinos. Asociación Argentina de Consorcio Regional Experimentación Agropecuaria (AACREA), Argentina. $25 \mathrm{pp}$.

de Lima, M. A. 2002. Metano de bovinos é monitorado. Jornal o Estado de São Paulo. 13 de noviembre. Brasil.

Ghida Daza, C. 2003. ¿Ganancia o sostenibilidad? Un análisis económico de estrategias agrícolas. Área Economía, Estadística e Informática. Estación Experimental Agropecuaria (EEA) Marcos Juárez. INTA. Argentina. 8 pp.

Hansen, M. N., S.G. Sommer, and K.Henriksen. 2002. Methane emissions from livestock manure - effects of storage conditions and climate. Greenhouse Gas Inventories for Agriculture in the Nordic Countries, Helsingør, Denmark. Pages 45-53. In: P. Søren, and O. Jørgen (eds.), DIAS Report, plant production 81. Danish Institute of Agricultural Sciences. Denmark.

IPCC. 1996. Guidelines for National Greenhouse Gas Inventories: Reference Manual. Intergovernmental Panel on Climate Change. Suiza. www.ipcc-nggip.iges.or.jp/public/public. htm. 76 pp. (Consultado en marzo de 2007).

Johnson, K.A., and D.E. Johnson. 1995. Methane emissions from cattle. J. Anim. Sci. 73:24832492.

Mangino,J.2003.USEPACattleEnteric Fermentation Model (CEFM). Non- $\mathrm{CO}_{2}$ Gases and Sequestration Branch. Environment Protection Agency (EPA), April 30. USA. 19 pp.

McGinn, S.M., Beauchemin, K. A., Coates, T., Colombatto, D. 2004. Methane emissions from beef cattle: Effects of monensin, sunflower oil, enzymes, yeast, and fumaric acid. J. Anim. Sci. 82:3346-3356.

Müller, B., U. Bartsch. 1999. The Modelling of Anthropogenic Methane Emissions: Methodology and Estimates. Oxford Institute for Energy Studies. Oxford, England. 35 pp.

Naredo, J.M. 1997. Sostenibilidad, diversidad y movilidad horizontal en los modelos de uso del territorio. http://habitat.aq.upm.es/cs/p2/a006. html. (Consultado en mayo de 2007).

Palliser, C.C., and S.L. Woodward. 2002. Using models to predict methane reduction in pasture-fed dairy cows. Proceedings Integrating Management and Decision Support. Coordinated by Susan M. Cuddy (CSIRO, Australia). Parte 1, vol. 482, p.162-167.

Sharma, C., A. Dasgupta, and A.P. Mitra. 2002. Inventory of GHGs and other urban pollutants from agriculture and waste sectors in Delhi and Calcutta. Proceedings of IGES/APN Mega-City Project. 6 pág. 23-25 January 2002. Kitakyushu, Japan.

Torroba, J.P. 1988. Invernada. Cuaderno de Actualización Técnica $\mathrm{N}^{\circ}$ 35. Asociación Argentina de Consorcios de Experimentación Agropecuaria (AACREA). Capital Federal. Argentina. 84 pp. 
Ulyatt, M.J., K.R. Lassey, I.D. Shelton, and C.F. Walker. 2002. Methane emission from dairy cows and wether sheep fed subtropical grassdominant pastures in midsummer in New Zealand. New Zealand Journal of Agricultural Research 45: 227-234

von Bernard, H. 2006. Sustentabilidad de la producción ganadera bovina. ¿Externalizar o internalizar los costos ambientales?. Un estudio en la pampa húmeda. Tesis para optar por el título de Master en Agronegocios y Alimentos. Escuela para Graduados Alberto Soriano. Facultad de Agronomía, Universidad de Buenos Aires. Buenos Aires, Argentina.

Westberg, H., B. Lamb, K.A. Johnson, and M. Huyler. 2001. Inventory of methane emissions from U. S. cattle. Journal of Geophysical Research 106:12633-12642. 